\title{
Study on polypropylene a c capacitors and its time dependence of loss tangent
}

\author{
Shivakumara swamy.R ${ }^{1}$ Dr.R Praksah ${ }^{2}$ Dr. K. N. Ravi ${ }^{3}$ \\ Ph.D Scholar, Dept. of EEE. Acharya Institute of Technology, Soldevanahalli, Bangalore - 560090 \\ Prof \& Head, Dept. of EEE. Don Bosco Institute of Technology Bangalore ${ }^{2}$ \\ Prof \& Head, Dept. of EEE. Saptagiri college of Engg Bangalore ${ }^{3}$
}

\begin{abstract}
Measurements are presented of the decrease of loss tangent with time of application of a high alternating stress and its return on removal of the stress. Measurements were made at an elevated temperature, as well as at ambient conditions. An explanation of the phenomena in terms of the

movement of charge carriers into the polymer and their recombination within the polymer is attempted.
\end{abstract}

Keywords: Dielectric materials, Organic insulating materials, Polymer.

\section{Introduction}

Although the ESR (equivalent series resistance) figure of a capacitor is mentioned more often, dissipation factor and loss tangent are also widely used and closely associated with the capacitor ESR.

Although dissipation factor and loss tangent are effectively the same, they take slightly different views which are useful when designing different types of circuit. Normally the dissipation factor is used at lower frequencies, whereas the loss tangent is more applicable for high frequency applications. The loss tangent is the tangent of the difference of the phase angle between capacitor voltage and capacitor current with respect to the theoretical 90 degree value anticipated, this difference being caused by the dielectric losses within the capacitor.

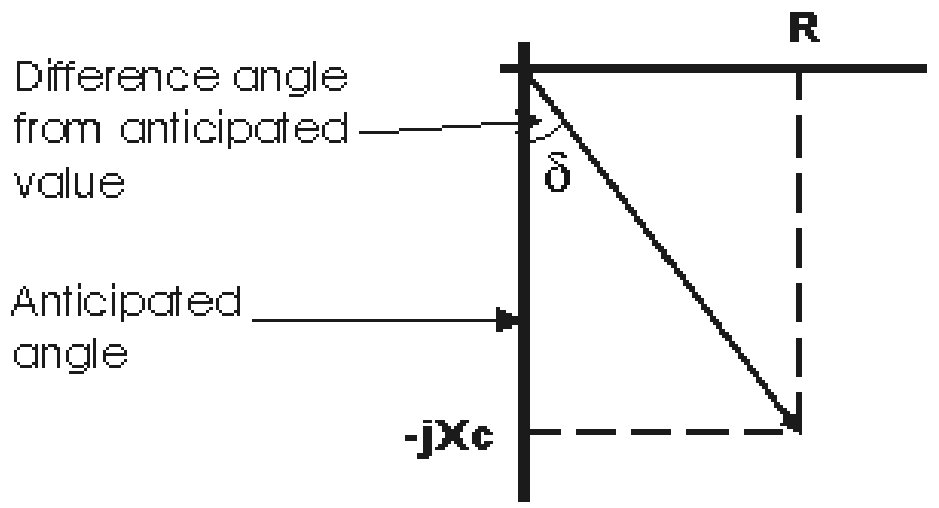

Fig.1: Capacitor loss tangent.

$\operatorname{Tan} \delta=\mathrm{ESR} / \mathrm{Xc}$

There are two deviations from simple linear dielectric behaviour to be found in liquid-impregnated capacitors. There is the well known Garton effect, ie, effectively a decrease in loss tangent with increasing applied measuring voltage, and there is a progressive fall in loss tangent during prolonged application of a high alternating stress. The latter effect is no so well documented, and we present some new experimental results as well as attempting an explanation of the phenomenon.

\section{Experimental details}

The capacitors investigated consisted of windings made with $6 \mu \mathrm{m}$ thick aluminium-foil electrodes and either 2-layer dielectrics of $2 \times 12.7 \mu \mathrm{m}$ polypropylene film or 3-layer dielectrics, where a paper layer $(10 \mu \mathrm{m}$ thick, density $1.0 \mathrm{~g} \mathrm{~cm}^{-3}$ ) was inserted between the two polypropylene films. The windings were flattened, and were vacuum- impregnated with epoxy-stabilised trichlordiphenyl ( t.c.d.p.) at a temperature of $60^{\circ} \mathrm{C}$. The 2 layer samples had a capacitance of $0.23 \mu \mathrm{F}$, the 3-layer ones $1 \mu \mathrm{F}$.

Capacitance and loss tangent measurements were made with an ampere-turns balance transformer bridge at $50 \mathrm{~Hz}$ using a tuned detector. The absolute accuracy of $\tan \delta$ measurement is estimated as $\pm 5 \times 10^{-5}$, and repeatability is $\pm 1 \times 10^{-5}$ 


\section{Measurement Results}

Fig. 2 shows loss tangent as a function of a.c. measuring stress at various times after the application of a stress of $50 \mathrm{MV} / \mathrm{m}$ (r.m.s.) to the sample, this stress only being interrupted briefly while making the measurements.

The sample containing paper as well as polypropylene showed similar characteristics (Fig. 3). In this case, the stress referred to is that in the polypropylene; the stress in the paper is lower by a factor of 2.7 (the permittivity ratio). Measurements were also made at $70^{\circ} \mathrm{C}$, where the phenomena were speeded up. In particular, the return of the losses towards the higher initial values on removing the electric stress can be observed within days at $70^{\circ} \mathrm{C}$, but takes months at ambient temperature.

The loss tangent at an arbitrary measuring stress of $10 \mathrm{MV} / \mathrm{m}$ is plotted against time in Figs. 4 and 5 (at $22^{\circ} \mathrm{C}$ in Fig. 4 and at $70^{\circ} \mathrm{C}$ in Fig. 5), with the samples on stress (50 MV/m) in Figs. $4 \mathrm{a}$ and $5 a$, and with the stress removed in Figs. $4 b$ and $5 b$.

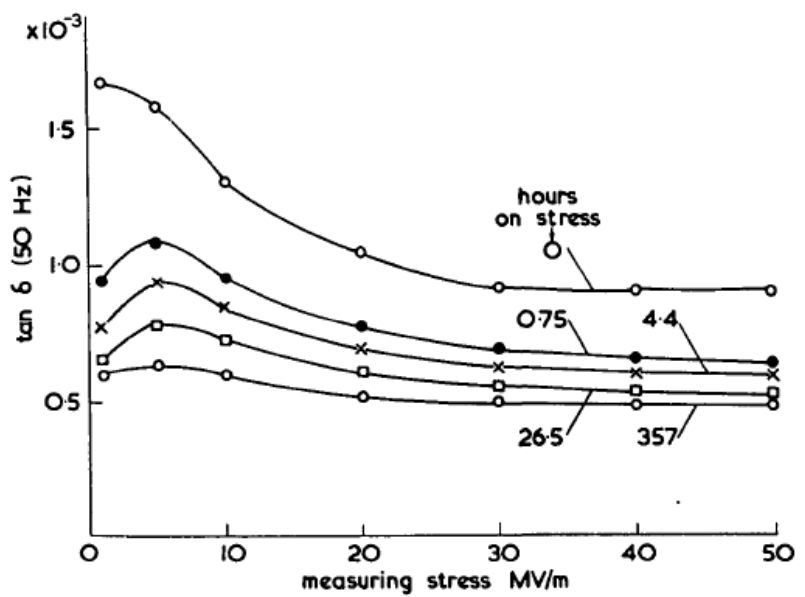

Fig. 2: Dissipation factor ( $\tan \delta$ ) of a 2-layer polypropylene capacitor after the application of an alternating stress of $50 \mathrm{MV} / \mathrm{m}$ for various periods.

Although only semi quantitative deductions are made from the results in this paper, for completeness it should be stated that the electrical/thermal history of the samples was as follows: Under stress at room temperature for $357 \mathrm{~h}$ (yielding Fig. $4 \mathrm{a}$ ); stressed for $24 \mathrm{~h}$ at $70^{\circ} \mathrm{C}$; left unstressed at $70^{\circ} \mathrm{C}$ (yielding Fig. $4 \mathrm{~b}$ ), stressed at $70^{\circ} \mathrm{C}$ (yielding Fig. 4a). Two different samples were stressed at $70^{\circ} \mathrm{C}$ for $72 \mathrm{~h}$ and at $2^{\circ} \mathrm{C}$ for $24 \mathrm{~h}$, and were then left unstressed at $22^{\circ} \mathrm{C}$ ielding Fig. $5 b$ ). In the time allowed in these easurements, the loss tangent did not fully recover its initial high value - neither at $22^{\circ} \mathrm{C}$ nor at $70^{\circ} \mathrm{C}$.

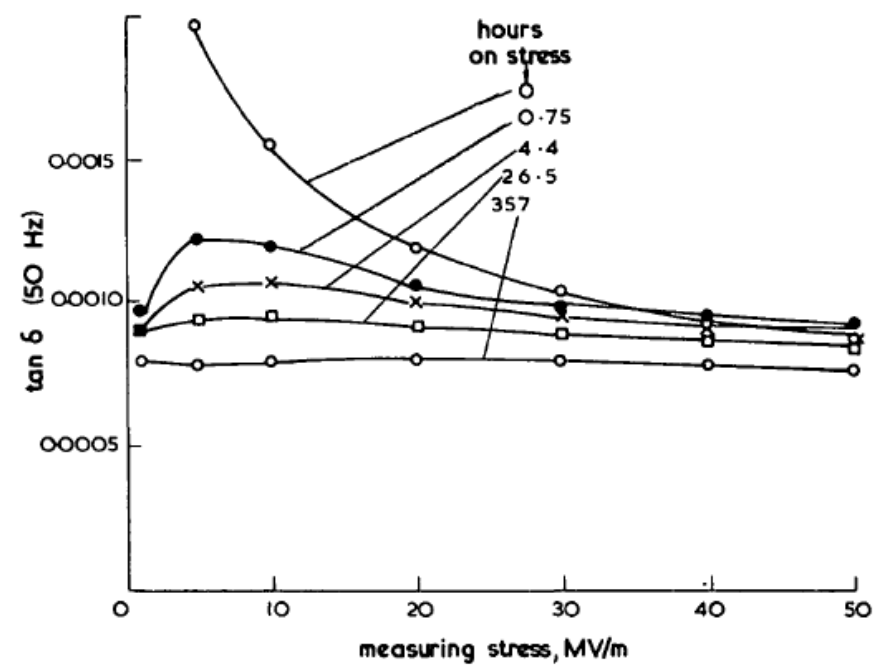

Fig.3:Loss tangents of a film-paper-film capacitor after the application of $50 \mathrm{MV} / \mathrm{m}$ (stress in film) for various periods. 

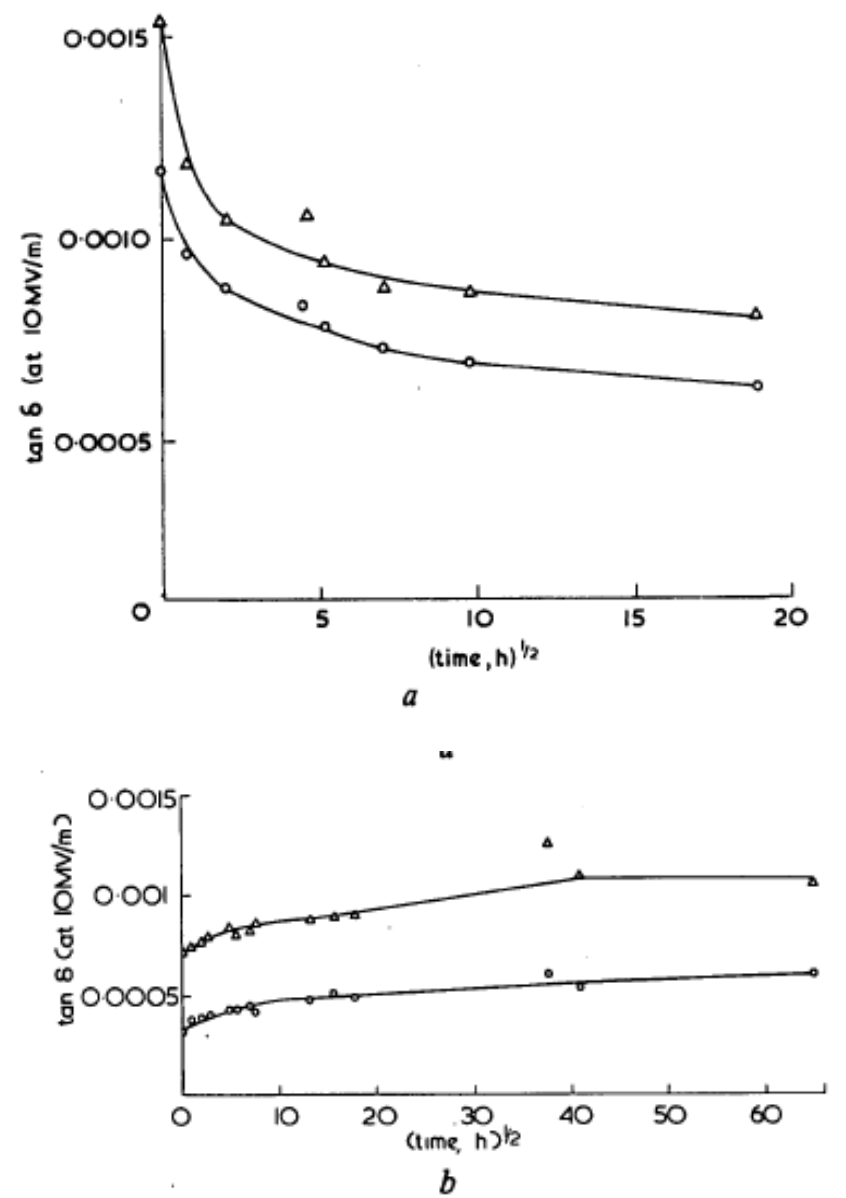

Fig. 4: Loss tangent, measured at $10 \mathrm{MV} / \mathrm{m}$, as a function of time.

Temperature $=22^{\circ} \mathrm{C}$

$a$ With samples under a stress of $50 \mathrm{MV} / \mathrm{m}$

1.film-paper-film

2. 2 layers polypropylene film

$b$ After removal of the stress

1. film-paper-film

2. 2 layers polypropylene film

\section{Discussion}

The phenomena shown can all be ascribed to variations in the loss tangent contributed by the liquid in the gaps between layers. This loss tangent is caused by conduction, any dipole losses in t.c.d.p. being very small at room temperature and above. The gaps are clearly of variable width, since the solid materials must be touching in many places, but an average value can be deduced from the fractional change in capacitance on impregnation. The total gap was $4.6 \mu \mathrm{m}$ for one of the 2-layer polypropylene specimens, i.e. $1.5 \mu \mathrm{m}$ per interface. There is an uncertainty of about $15 \%$ because of swelling of the polymer film in the impregnate. The gaps for the 3-layer samples are expected to be similar.

The Garton-effect curves in Fig. 2show a pronounced maximum at a field of $5 \mathrm{MV} / \mathrm{m}$ (except for the initial curve), and this allows a mobility $(\mu)$ of the charge carriers to be derived, using the above gap width. Garton's equation, slightly rearranged, is $\mu=\omega d / 2 A_{0}$ where $d$ is the gap width and $A o$ the amplitude of the alternating field (angular frequency $\varpi$ ). The result is $\mu=10^{-2} \mathrm{~m} 2 / \mathrm{Vs}$. A simple calculation of overall loss tangent in terms of the individual layers' permittivity and losses allows a value of 0.0010 for the capacitor (at low field, on the $0.75 \mathrm{~h}$ curve in Fig. 2) to be translated into a value of 0.013 for the impregnant. The volume concentration of charge carriers can then be deduced, assuming that each carries one electronic charge: $\omega$ 
$n=\sigma / e \mu=10^{19} m^{-3}(\sigma=$ conductivity $)$
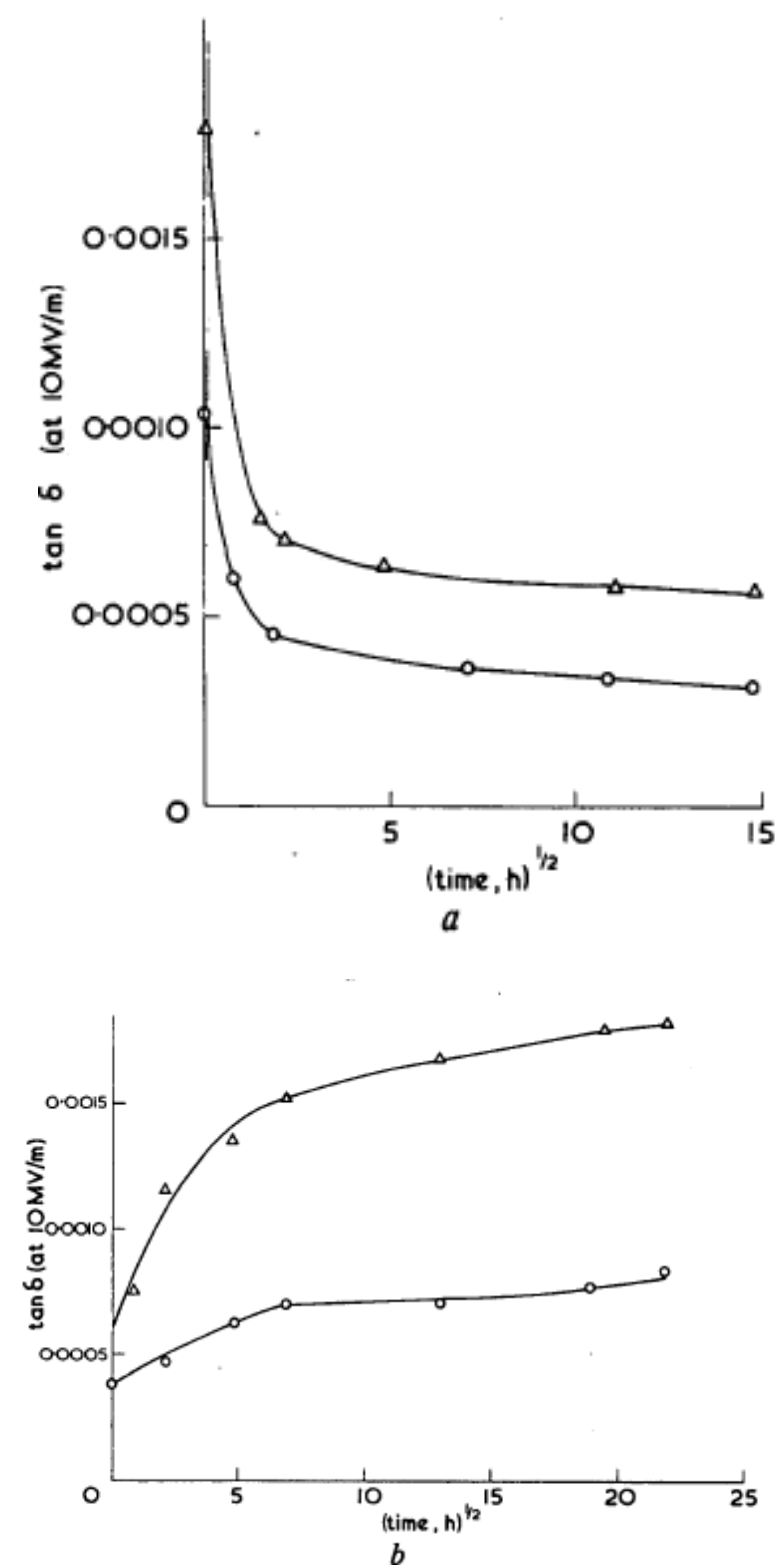

Fig. 5: Loss tangent, measured at $10 \mathrm{MV} / \mathrm{m}$, as a function of time

Temperature $=70^{\circ} \mathrm{C}$

$a$ With samples under a stress of SO MV/m

1 film-paper-film

2. 2 layers of polypropylene film

$b$ After removal of the stress

1. film-paper-film

\section{2 layers of polypropylene film}

The charge carriers are likely to be sizable entities, a Stoke's law calculation of equivalent sphere diameter yielding $2 * 5 \mathrm{~nm}$. Use of the word 'ion' to denote them in the following paragraphs does not imply an ionised atom, but is just a shorter word.

It is known from subsidiary tests that, to induce a loss tangent of 0.013 in t.c.d.p. at room temperature, approximately 10 parts in $10^{6}$ of an amine impurity must be added. This corresponds to a molecular concentration of $5 \times 10^{22} \mathrm{~m}^{-3}$. Although the species of impurity causing the effects described in the capacitors is not known, it is most unlikely to be so much more 'potent' that a concentration 5000 times weaker could lead to the same conductivity; hence the impurity is only ionised to a very small degree. This is consistent with the 
occurrence of a peak in the Garton curve and with the fact that the tan-5/field curve does not follow an $(\mathrm{E})^{-3 / 2}$ law.

The gradual disappearance of the Garton effect along with the general reduction in loss tangent demonstrates that this 'a.c. cleanup' is caused by the ions in the liquid becoming immobilised; They could be immobilised by reaction with the electrodes, and such effects are encountered with liquid test cells,s but one would not expect such reactions to take weeks. Also, the present work uses such high stresses that the electrodes are much more likely to generate ions than to immobilise them.6 It is therefore assumed that the ions are immobilised in the polymer films, and it is the mechanism of this process that needs some explanation.

There is no question that impurities can move in polypropylene. The film is swollen by t.cd.p., and it is well known that impurities can leach out of the film, raising the loss tangent of the t.c.d.p. At $100^{\circ} \mathrm{C}$, the leaching process is complete in 2-3 days, and curves of Fig. $4 b$ are consistent with this time scale, the return of the high loss tangent in the absence of the field being due to the diffusion of impurities back into the t.c.d.p. from the polymer films. An idealised diffusion process, the mobile species going from a slab of thickness 21 to a medium of (permanently) negligible concentration, would yield a straight line followed by a rapid changeover to saturation on ${ }^{\wedge}$ plot of (number of impurities transferred) against (time) ${ }^{1 / 2}$. In our case, the capacitor loss tangent should be a measure of the number of impurities transferred to the liquid, and it will be noticed that the time scale is given in (hours) ${ }^{1 / 2}$. There are two obvious reasons why the curves are much smoother than was expected from the ideal case:

(a) There will be a whole spectrum of impurities with different diffusion constants.

(b) The liquid in the gap does not represent a medium of negligible concentration, and the approach to an equilibrium partition o"f impurities between solid and liquid will be more gradual than in the ideal case.

A saturation region in the curves of Fig. $4 b$ can be recognised nevertheless, and this yields an approximate average diffusion constant $/$ ) $=I^{2} / T=0.5 \times 10^{-15} \mathrm{~m}^{2} / \mathrm{s}$, where $\mathrm{T}$, the time at the beginning of saturation, has been taken as $22 \mathrm{~h}$. Provided the impurities, when ionised, move in the same way, their mobility in the swollen polypropylene $\left(\right.$ at $70^{\circ} \mathrm{C}$ ) can be calculated via the relation $\mathrm{kT} / \mathrm{e}=D / \mu$ as $\mu=2 \times 10^{-14} \mathrm{~m}^{2} / \mathrm{s}$

Turning now to the cleanup effect when the high alternating field is present, consider the liquid film bounded by two polypropylene faces. During each halfcycle of the field, the ions, after crossing the gap, will be drawn a certain way into the polymer, and, during the succeeding halfcycle, will be drawn out again, will cross the gap and will penetrate the opposite faces. Assuming for the moment that the ions move virtually as one block (they are swept across the gap in a small fraction of a cycle) and since both the geometry and the applied field are symmetrical, the ions of opposite polarity should both cross the gap at the time of maximum field and achieve maximum penetration (of the order of $10 \mathrm{~nm}$ for calculated mobility and field applied) at zero field. No cleanup should be observed, since there is nothing to hold the ions in the polymer and they still cross the gap every halfcycle.

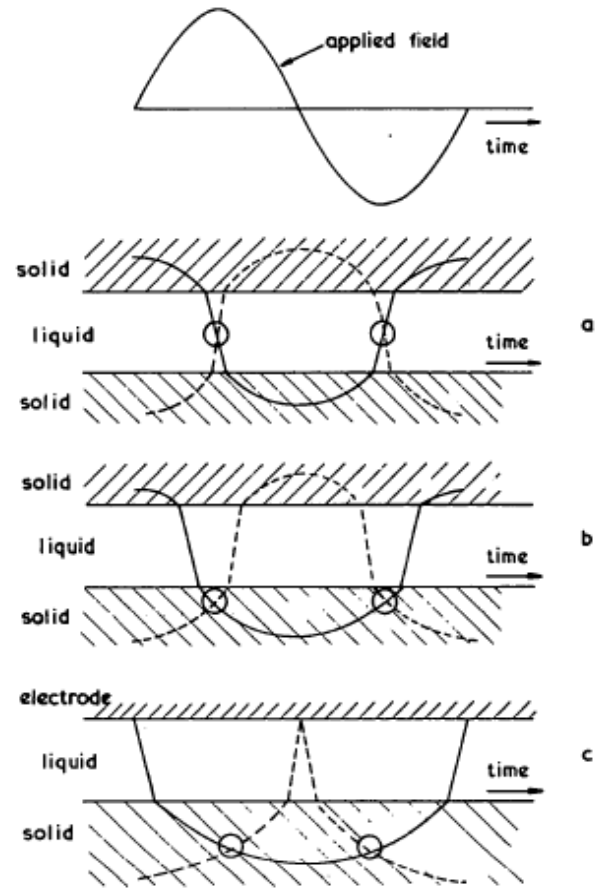

Fig. 6: Schematic illustration of the paths taken by a positive ion and a negative ion during one cycle of the applied field. 
a. Symmetrical paths at a liquid gap between two films

b. Asymmetrical paths at a similar gap.

c. Paths at a gap between film and aluminium electrode.

In our view, the crucial point is that ions of opposite polarity should encounter each other within the polymer, because the neutral molecules formed by recombination of these ions would not easily reionise, being situated in a medium of lower permittivity than that of the impregnant.

The opportunity for such encounters will, in fact, arise because the assumption of perfect symmetry, made above, is not fully justified. There are various reasons why the ions will not move as a coherent layer. There is certainly random scattering, which can delay some ions and speed others, and there is the possibility of further ionisation of impurities inthe liquid phase. The time of 'birth' of a fresh ion pair would be random with respect to the phase of the applied field. An ion can remain in a geometrically asymmetric path without any forces tending to return it to a more symmetrical path. With random paths, there will still be ions of one polarity within the polymer, on their way out, when ions of opposite polarity are arriving on their way in. Recombination will take place rendering the ions immobile. Fig. 6 illustrates the point.

The case of the liquid gap bounded by a metal electrode on one side and by a polymer film on the other is rather simpler. Since the electrode is inert, ions of a given sign accumulate there in one halfcycle and form a layer of thickness equal to the Debye length (about $0.5 \mu \mathrm{m}$ in our case). At the beginning of the reverse half cycle, they cross the gap and are drawn into the polymer film, while the ions of opposite sign are still in the process of emerging from the film. As seen in Fig. 5c, the ions spend most of their time within the polymer and very little time at the electrode. Thus again, recombination can take place within the polymer film.

The form of the $\tan \delta /$ time curves during the cleanup is The form of the tan-5/time curves during the cleanup is related to the ion recombination rate, rather than to iffusionrate as it was during the deterioration process with the field removed. The neutral molecules formed within the surface layers of the polypropylene do diffuse away towards the interior of the solid - otherwise the cleanup would be very short-lived - but the measured tan 5 is, of course, related to the number of ions remaining in circulation. Their rate of recombination within the solid would be difficult to estimate theoretically.

The position at a boundary with paper may be different. Paper is known to be an active absorptive agent, and it tends to improve contaminated t.c.d.p. even without a field being applied. An increased ion concentration at the surface caused by the application of the field may induce the cellulose to absorb further ions by a shift in the absorption/ desorption equilibrium.

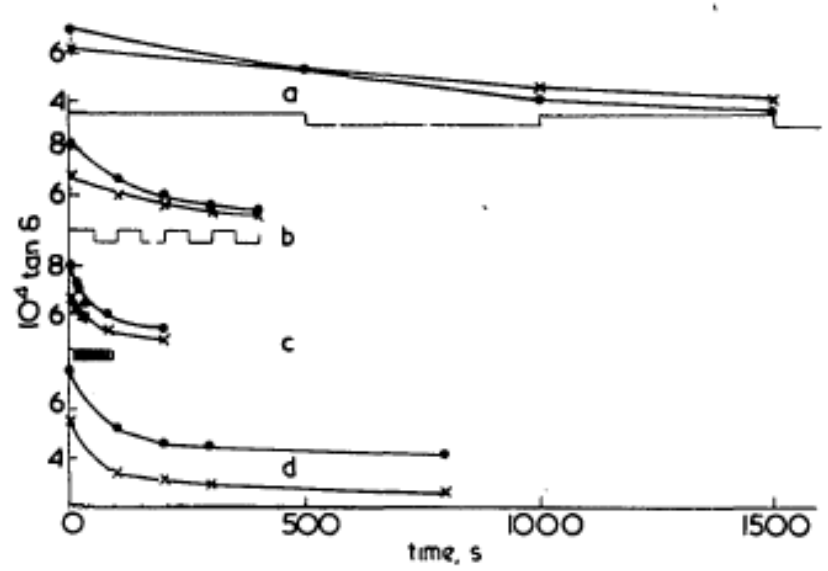

Fig. 7: Loss tangents (measured at 10MV/m)for two similar 2- layer polypropylene capacitors as a function of time of stressing, for square-wave alternating fields of long period,contrasted with the effect of a $50 \mathrm{~Hz}$ field . Temperature $=70^{\circ} \mathrm{C}$

(a) $50 \mathrm{MV} / \mathrm{m}$ d.c. reversed every $500 \mathrm{~s}$

(b) $50 \mathrm{MV} / \mathrm{m}$ d.c. reversed every $50 \mathrm{~s}$

(c) $50 \mathrm{MV} / \mathrm{m}$ d.c. reversed every $5 \mathrm{~s}$

(d) $50 \mathrm{MV} / \mathrm{m}$ (r.m.s.) a.c, $50 \mathrm{~Hz}$

The mechanism described depends on frequent field reversals to bring positive and negative ions to recombine and become immobilised. On the other hand, the application of a direct field could draw the ions much further into the polymer, and they would not need to be given time to diffuse away from the boundaries. A few field reversals might suffice to immobilise the ions. Some short term tests were carried out on these ideas. Fig. 6 shows the results for direct fields reversed every 500, every 50 and every 5 seconds. For comparison, the 
effect of a $50 \mathrm{~Hz}$ alternating field over a similar time is also shown. Clearly, the more frequent reversals accelerate the decrease in loss tangent, which is consistent with the mechanism proposed.

\section{Conclusions}

The loss tangent of liquid-impregnated polypropylene- film capacitors decreases with time on alternating stress. The process is faster at elevated temperature. Like the Garton effect, it is caused by the immobilisation of charge carriers present in the liquid phase of the dielectric. A mechanism is proposed that involves the field bringing the ions a little way into the solid; reversal of the field ensures that the ions recombine within the solid, and the neutral molecules, which no longer contribute to the losses, then diffuse into the bulk of the solid.

\section{Acknowledgements}

We would thank Dr.Shivakumara Aradya, Director (retired), CPRI Bangalore, for their numerous suggestions and helpful discussions. Skilful technical assistance was provided by Mr.Jaganath, Managing Director M/S Jognics super capacitors ltd Bangalore.

\section{References}

[1]. C.W.Reed, S.W.Cichanowski, "The fundamentals of ageing in HV Polymer-film Capacitors", IEEE Transactions on Dielectrics and Electrical Insulation, Vol. 1, No. 5, Oct 1994

[2]. H.N.Nagamani, S.N.Moorching, "A study on the influence of partial discharges on the life of composite dielectric capacitors", Proceedings of the 5Ih International Conference on Properties and Applications of Dielectric Materials, May 1997. Seoul, Korea.

[3]. C.A.Nucci, S,Pirani, M.Rinaldi, "Pulse withstand capabitity of self-healing Metalized Polypropylene capacitors in power applications", IEEE Transaction on Electrical Insulation, Vo1.26, No.1, Feb 1991

[4]. $\quad$ B. Sanden and E. Ildstad, "DC electrical and mechanical characterisation of polypropylene film", IEEE Intern. Conf. Sol. Dielectr. (ICSD), Västerås, Sweden, pp. 210-213, 1998. 